\title{
Incidence of antipsychotic use in relation to diagnosis of Alzheimer's disease among community-dwelling persons
}

Marjaana Koponen, Anna-Maija Tolppanen, Heidi Taipale, Antti Tanskanen, Jari Tiihonen, Kristina Johnell, Johan Fastbom, Riitta Ahonen and Sirpa Hartikainen

\section{Background}

Behavioural and psychological symptoms of dementia are frequently treated with antipsychotics.

\section{Aims \\ To determine the incidence of antipsychotic use in relation to diagnosis of Alzheimer's disease.}

\section{Method}

Cohort of all community-dwellers in Finland diagnosed with Alzheimer's disease in 2005 and matched controls. All antipsychotics dispensed between 1995 and 2009 were extracted from the Finnish National Prescription Register.

\section{Results}

Altogether 1996/6087 (32.8\%) persons with Alzheimer's disease initiated antipsychotic use. The incidence of antipsychotic use was fivefold among persons with
Alzheimer's disease compared with controls, started to increase 2-3 years before diagnosis and was highest during the first 6 months after diagnosis.

\section{Conclusions}

A distinct increase in antipsychotic initiations occurs in the same time window as Alzheimer's disease diagnosis.

\section{Declaration of interest}

J.T. received personal fees from AstraZeneca, Janssen-Cilag, Otsuka, Bristol-Myers Squibb, Lundbeck, Organon, Eli Lilly, F. Hoffmann-La Roche, GlaxoSmithKline, Pfizer, Novartis and receives a grant from Stanley Foundation, outside the submitted work.

\section{Copyright and usage}

(c) The Royal College of Psychiatrists 2015.
Up to $90 \%$ of persons with dementia experience behavioural and psychological symptoms of dementia (BPSD) at some point during the course of the disease. ${ }^{1,2}$ BPSD include symptoms such as apathy, depression, anxiety, agitation, aggression, delusions, hallucinations, sleep disturbances, euphoria and disinhibition. Antipsychotics are frequently used to treat BPSD among persons with Alzheimer's disease and other dementias, especially in institutional settings. ${ }^{3-5}$ However, antipsychotic use has been associated with mortality ${ }^{6-8}$ and serious adverse drug events including extrapyramidal symptoms, ${ }^{9}$ hip fracture ${ }^{10}$ and stroke $e^{11,12}$ among older people with dementia. In addition, antipsychotics may accelerate cognitive decline. ${ }^{13-15}$ According to systematic reviews and meta-analyses certain antipsychotic drugs have efficacy for specific BPSD including psychosis and aggression. ${ }^{16-19}$ However, improvements on rating scales for BPSD have been modest and not all randomised controlled trials have been able to demonstrate beneficial effects. As the risks of antipsychotics may outweigh their benefits among persons with Alzheimer's disease, guidelines recommend that antipsychotics should be used only in the treatment of severe psychotic symptoms, aggression and agitation when the symptoms cause significant distress or risk of harm to the patient or others. ${ }^{20-22}$ There are no previous studies on when antipsychotics are prescribed for the first time in the course of Alzheimer's disease. We describe the incidence of antipsychotic use up to 8 years before and 4 years after diagnosis of Alzheimer's disease among community-dwelling persons.

\section{Method}

This study is based on a nationwide register-based cohort study MEDALZ-2005 (Medication and Alzheimer's disease) described in detail elsewhere. ${ }^{3,23}$ Briefly, the MEDALZ-2005 included all community-dwelling persons diagnosed with Alzheimer's disease residing in Finland on 31 December $2005(n=28093)$ and one age-, gender- and region of residence-matched person without Alzheimer's disease. Clinically verified Alzheimer's disease diagnoses were identified from the Special Reimbursement Register maintained by the Social Insurance Institution of Finland (SII). This register contains records of persons who are entitled for higher reimbursement due to chronic diseases such as Alzheimer's disease, diabetes and epilepsy. To be entitled for reimbursement, a patient must meet predefined criteria and a diagnosis statement must be submitted to the SII for approval. The SII requires that the medical statement verifies that the patient has: (a) symptoms consistent with Alzheimer's disease; (b) experienced a decrease in social capacity over a period of at least 3 months; (c) received a computed tomography/magnetic resonance imaging scan; (d) had possible alternative diagnoses excluded and (e) received confirmation of the diagnosis by a registered geriatrician or neurologist. The SII reviews all medical statements and checks that the diagnosis of Alzheimer's disease is based on the National Institute of Neurological and Communicative Disorders and Stroke and the Alzheimer's Disease and Related Disorders Association $^{24}$ and DSM-IV ${ }^{25}$ criteria for Alzheimer's disease. This study was restricted to those persons diagnosed with Alzheimer's disease in $2005(n=7217)$ and their matched control persons. Data linkage was performed by the SII and they de-identified the data. According to Finnish legislation, no ethics committee approval was required because only de-identified register-based data were used and the study participants were not contacted.

Data on all antipsychotics dispensed between 1995 and 2009 were extracted from the Finnish National Prescription Register for each study participant with and without Alzheimer's disease. The Prescription Register contains records of all reimbursed drug purchases of Finnish residents living in non-institutional settings. 
All drugs in the Prescription Register are categorised according to the World Health Organization (WHO) Anatomical Therapeutic Chemical (ATC) Classification system. ${ }^{26}$ The rate of new antipsychotic users (ATC code N05A, excluding lithium and prochlorperazine) per 100 person-years was calculated for every 6 months up to 8 years before and 4 years after Alzheimer's disease diagnosis. A 2-year washout period was applied starting 10 years before Alzheimer's disease diagnosis. A new user was defined as a person who had no antipsychotic purchases during the washout period but had at least 1 antipsychotic purchase during the 12-year follow-up. The index date for the matched control person without Alzheimer's disease was defined as the date of Alzheimer's disease diagnosis of the person with Alzheimer's disease.

Data on history of schizophrenia, schizotypal and delusional disorders (ICD-10 ${ }^{27}$ codes F20-29; ICD- ${ }^{28}$ codes 295, 297, 298, 3010 and 3012; ICD-8 ${ }^{29}$ codes 295, 297, 298, 29999, 30100 and 30120) and bipolar disorder (ICD-10 codes F30-31; ICD-9 codes 2962, 2963, 2964 and 2967; ICD-8 codes 29610, 29620, 29630, 29688 and 29699) were collected from the Hospital Discharge Register (data available since 1972). Only diagnoses recorded before diagnosis of Alzheimer's disease or index date for the controls were included as history of schizophrenia, schizotypal and delusional disorders or bipolar disorder. Persons with history of schizophrenia, schizotypal and delusional disorders or bipolar disorder were excluded with their matched pairs $(n=298)$ to remove the effect of these diagnoses on antipsychotic initiation.

A modified Charlson comorbidity index score ${ }^{30}$ was calculated for each person at the time of Alzheimer's disease diagnosis/index date and for each antipsychotic initiator at the time of first purchase. Comorbidity score was calculated using the following diseases with corresponding scores. Score of 1: coronary artery disease, heart failure, type 1 or 2 diabetes, chronic asthma or chronic obstructive pulmonary disease, rheumatoid arthritis and disseminated connective tissue diseases. Score of 2: severe renal failure and all cancers. Data on comorbidities were extracted from the Special Reimbursement Register. Use of antidepressants (N06A) and use of benzodiazepines (N05BA, N05CD) and related drugs (N05CF) were assessed at the time of Alzheimer's disease diagnosis/index date (when comparing persons with and without Alzheimer's disease) and for each antipsychotic user at the time of initiation (when comparing users with and without Alzheimer's disease). Data on history of diagnosed depression (ICD-10 codes F32-39; ICD-9 codes 2961, 2968, 3004 and 3011; ICD-8 codes 29600, 30040, 30041 and 30110) were collected from the Hospital Discharge Register. Only diagnoses recorded before the time of Alzheimer's disease diagnosis/index date and for each antipsychotic user before the time of initiation were considered in the comparisons.

As the Prescription Register does not cover drugs used in nursing homes and hospitals, the date of long-term institutionalisation for each participant during the follow-up was obtained from the SII. In addition, hospital stays of more than 90 days were extracted from the Hospital Discharge Register maintained by the National Institute of Health and Welfare. Follow-up was censored at the start of long-term institutionalisation/hospitalisation, death or end of the study period, whichever occurred first. The formation of the study sample and reasons for exclusion and censoring are summarised in Fig. 1. Altogether, 33 matched controls had temporary reimbursement for Alzheimer's disease before 2006 and 511 were diagnosed with Alzheimer's disease during 2006-2009. These persons with their matched pairs were excluded from the analysis. In addition, pairs were excluded if either matched person with or without Alzheimer's disease had a history of schizophrenia, schizotypal and delusional disorders or bipolar disorder, used antipsychotics during the 2-year washout period or were in long-term institutional care at the start of follow-up. The final study sample consisted of 6087 matched pairs $(n=12174)$. The mean age of the study sample at the time of

Table 1 Description of the study sample and initiators of antipsychotic use

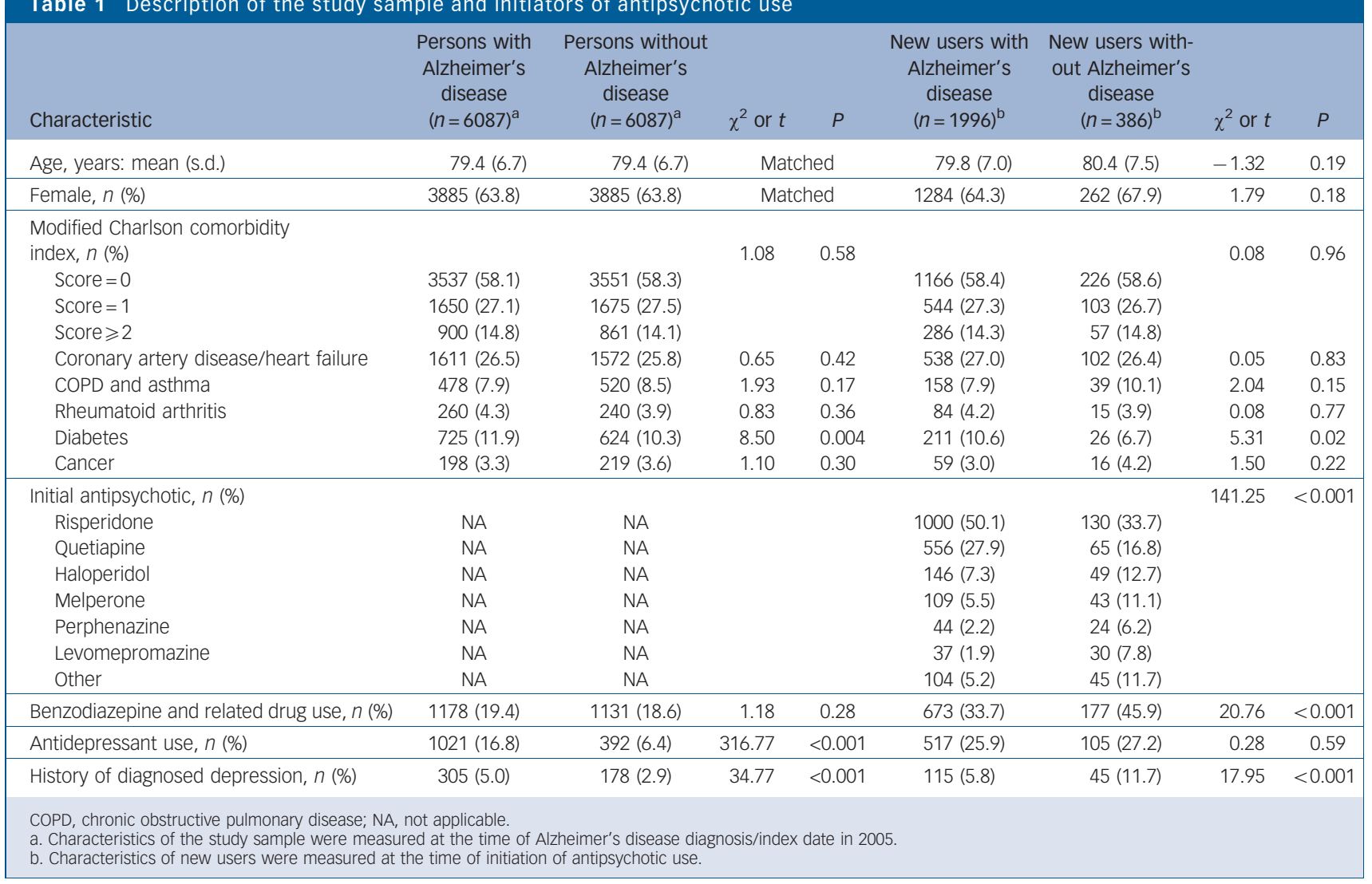




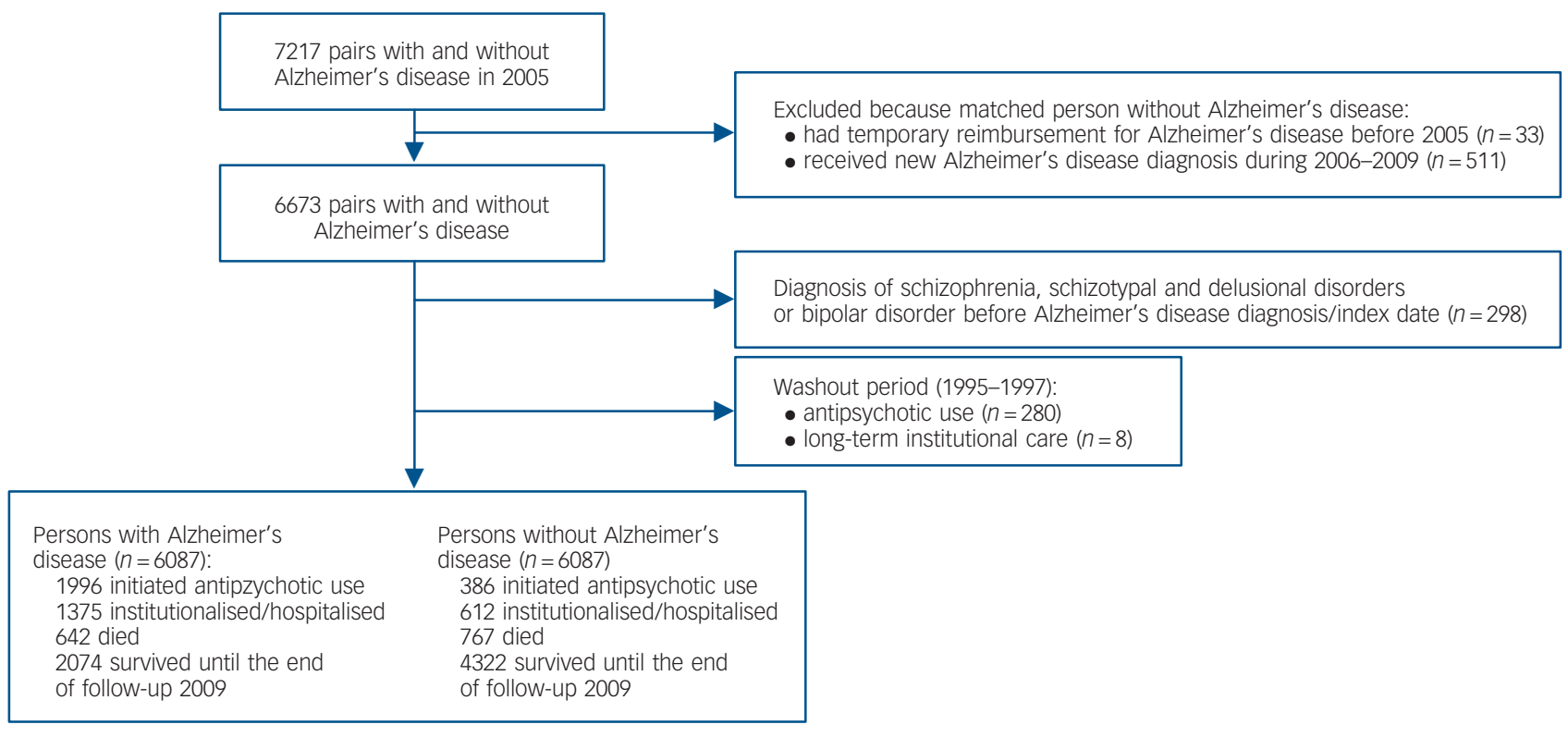

Fig. 1 Flow diagram of the study sample.

Alzheimer's disease diagnosis was 79.4 years (range 42-97) and $63.8 \%$ were female (Table 1). The age, gender and regional distribution of the final sample $(n=12174)$ did not differ from the initial study sample $(n=14434)$.

\section{Statistical analyses}

Characteristics of persons with and without Alzheimer's disease were compared using $\chi^{2}$-test for categorical variables and $t$-test for continuous variables. Poisson regression was used to compute incidence rate ratios (IRRs) and 95\% confidence intervals for every 6-month period to estimate the difference in incidences of antipsychotic use between persons with and without Alzheimer's disease. Logistic regression was used to analyse the relationship between initiation of antipsychotic use before Alzheimer's disease diagnosis and age and gender. All analyses were performed with SAS (Version 9.3; SAS Institute, Cary, North Carolina, USA).

\section{Results}

During the follow-up, 1996 (32.8\%) persons with Alzheimer's disease initiated antipsychotic use (Fig. 1). The incidence of new users was five times higher (IRR $=5.17$; 95\% CI 4.64-5.77) among persons with Alzheimer's disease compared with the matched persons without Alzheimer's disease, of which 386 (6.3\%) initiated antipsychotic use.

At the time of Alzheimer's disease diagnosis (index date), a higher proportion of persons with Alzheimer's disease had history of diagnosed depression and diabetes, and were antidepressant users compared with persons without Alzheimer's disease (Table 1). There were no differences in the mean age, gender distribution and comorbidities between users with and without Alzheimer's disease at the time of initiation of antipsychotic use. New antipsychotic users without Alzheimer's disease used benzodiazepines and related drugs more frequently $(45.9 \%)$ than users with Alzheimer's disease $(33.7 \%, P<0.001)$. A higher proportion of antipsychotic users without Alzheimer's disease had history of diagnosed depression $(11.7 \%)$ compared with new users with Alzheimer's disease $(5.8 \%, P<0.001)$. A fourth of both new users with and without Alzheimer's disease used antidepressants at the time of initiation of antipsychotic use. The profile of antipsychotics prescribed was different between persons with and without Alzheimer's disease $(P<0.001)$. Among persons with Alzheimer's disease use was initiated most frequently with risperidone $(50.1 \%, 1000 / 1996)$ or quetiapine $(27.9 \%, 556 /$ 1996). Similarly among new users without Alzheimer's disease, the most prevalent antipsychotic was risperidone $(33.7 \%, 130 /$ $386)$ followed by quetiapine $(16.8 \%, 65 / 386)$. Other antipsychotics comprised 22\% of initiations for persons with Alzheimer's disease and $49 \%$ for persons without Alzheimer's disease.

The rate of new users among persons with Alzheimer's disease significantly increased 2-3 years before Alzheimer's disease diagnosis compared with the rate among the controls without Alzheimer's disease (IRR $=2.09$; 95\% CI $1.05-4.16$ for time period of -3 to -2.5 years before diagnosis; Fig. 2). The incidence of antipsychotic use was highest during the first 6 months after Alzheimer's disease diagnosis (13.9 new users per 100 personyears) and remained at a high level thereafter (8.6-11.3 new users per 100 person-years). However, the incidence of antipsychotic use among the controls without Alzheimer's disease remained stable during the 12-year follow-up ranging between 0.2 and 1.3 new users per 100 person-years. Among antipsychotic users with Alzheimer's disease, $71.5 \%$ of the use was initiated at the time of diagnosis and thereafter, whereas among the controls without Alzheimer's disease $44.8 \%$ of antipsychotic use was initiated after and $55.2 \%$ before the index date $(P<0.001)$.

A higher proportion of those who were aged 80 years or over at the time of diagnosis (33.4\%) had initiated antipsychotic use before diagnosis compared with those aged less than 80 years (23.1\%; OR $=1.64$ 95\% CI 1.34-2.00 adjusted for gender). Of women with Alzheimer's disease, 30.1\% initiated antipsychotic use before Alzheimer's disease diagnosis in comparison to $25.6 \%$ of men with Alzheimer's disease $(P=0.033)$. However, in a logistic regression analysis adjusted for age, gender was not significantly associated with initiating antipsychotic use before diagnosis $(\mathrm{OR}=1.16$; 95\% CI 0.94-1.43).

\section{Discussion}

\section{Principal findings}

The incidence of antipsychotic use started to increase among persons with Alzheimer's disease already 2-3 years before 


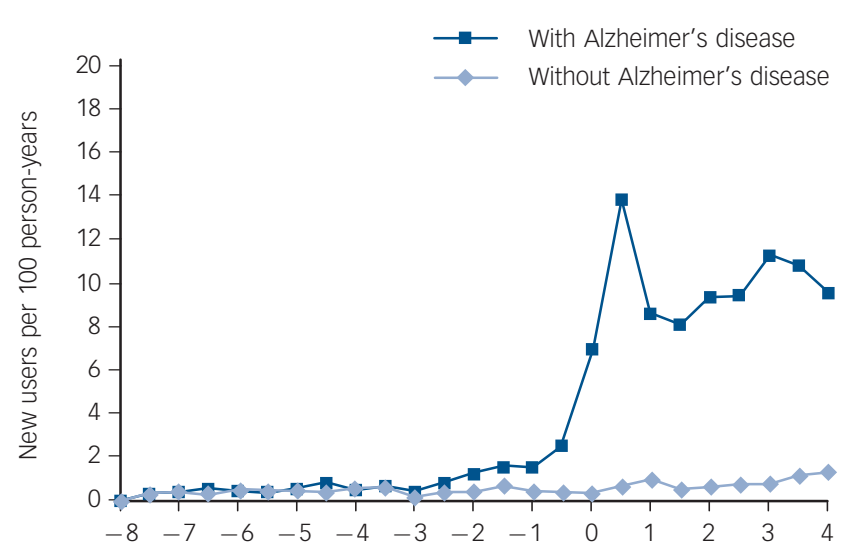

Time in years before and after the date of Alzheimer's disease diagnosis

Fig. 2 Incidence of antipsychotic use in relation to diagnosis of Alzheimer's disease among persons with and without Alzheimer's disease.

The date of Alzheimer's disease diagnosis of the person with Alzheimer's disease was defined as the index date (point zero) for the matched control person without Alzheimer's disease.

Alzheimer's disease diagnosis which might indicate early behavioural and psychological symptoms of Alzheimer's disease. Behavioural and psychological symptoms are frequent in persons with mild cognitive impairment and these might be one of the earliest symptoms of Alzheimer's disease in some persons., ${ }^{1,31}$ Thus, behavioural and psychological symptoms in the prodromal phases of Alzheimer's disease could explain the increased use of antipsychotics before the diagnosis. On the other hand, the increased use of antipsychotics before the diagnosis of Alzheimer's disease could partly result from the treatment of delirium. Based on previous research, delirium increases the risk for being diagnosed with dementia. ${ }^{32,33}$ In addition, delirium increases cognitive decline and might be a risk factor for new-onset dementia. ${ }^{34}$ As the neuropathological process of Alzheimer's disease begins decades before symptoms' onset and fulfilment of diagnostic criteria for Alzheimer's disease, ${ }^{35}$ the observed increase in the incidence of antipsychotic use 2-3 years before diagnosis could not have increased the risk of Alzheimer's disease. However, antipsychotic use might have accelerated the emergence and exacerbation of symptoms by declining cognition. ${ }^{14,15}$

The majority (72\%) of antipsychotic use was initiated at the time of Alzheimer's disease diagnosis and thereafter. This is likely to be related to the increased incidence of BPSD and delirium after the diagnosis. In addition, the threshold of prescribing antipsychotics for older people might be lower after the diagnosis of Alzheimer's disease, although guidelines recommend cautious and limited use. ${ }^{20-22}$ The highest rate of new users was observed during the first 6 months after Alzheimer's disease diagnosis. The finding of distinct increase in incidence of use 6 months before and after Alzheimer's disease, diagnosis is similar to results of Martinez et $a l^{36}$ who found a sharp increase in prevalence of antipsychotic use around the time of diagnosis among community-dwelling persons with dementia in the UK.

Those aged 80 years or over at the time of diagnosis were more likely to have initiated antipsychotic use before diagnosis compared with those aged less than 80 years. This may indicate that persons who were aged 80 or above at the time of diagnosis were more likely to have BPSD requiring antipsychotic use before diagnosis. On the other hand, physicians may have been more likely to treat these persons symptom-based with antipsychotics and the diagnosis might have been delayed. Although men with Alzheimer's disease are more likely to use more than one antipsychotic concomitantly and use antipsychotics with higher doses than women, ${ }^{37,38}$ a higher proportion of women initiated antipsychotic use before Alzheimer's disease diagnosis. However, this association did not persist after adjusting for age.

\section{Rationality of antipsychotic prescribing}

The Finnish current care guideline recommends that all persons with Alzheimer's disease should be treated with acetylcholinesterase inhibitors and/or memantine unless there is a specific contraindication. ${ }^{21}$ The highest rate of new antipsychotic users occurred directly after Alzheimer's disease diagnosis which is also the time when acetylcholinesterase inhibitor therapy is initiated and its dose is optimised. As antipsychotics may further impair cognitive function, ${ }^{14,15}$ the rationality of initiating antipsychotic use when trying to obtain the best response to acetylcholinesterase inhibitor therapy is questionable. In addition to poor cognitive function, ${ }^{14,15}$ antipsychotic use has been associated with increased mortality and serious adverse drug events like strokes, extrapyramidal symptoms and hip fractures ${ }^{6-12}$ which warrants careful weighing of benefits and risks for the individual patient.

The Finnish current care guideline on memory disorders recommends antipsychotic use only for the most severe BPSD including severe aggression and psychotic symptoms. ${ }^{21}$ It is also stated that antipsychotics should not be used in the treatment of BPSD for which they are not efficacious for, including hoarding, wandering, yelling, sexual disinhibition and eating inedible objects. We do not know whether physicians prescribe antipsychotics according to these recommendations in clinical practice. The high rate of antipsychotic use among persons with Alzheimer's disease observed in this study suggests otherwise. It seems that antipsychotics are not prescribed only for severe aggression and severe psychotic symptoms but also for other behavioural and psychological symptoms without evidence of benefits. However, some of the observed rate of antipsychotic use among persons with Alzheimer's disease could be due to delirium as dementia is a major risk factor for delirium. ${ }^{39}$

Of the controls without Alzheimer's disease 6\% initiated antipsychotic use during the follow-up. The Prescription Register data did not include indications for antipsychotic use. At the time of initiation of antipsychotic use, a higher proportion of controls without Alzheimer's disease used benzodiazepines and related drugs and had history of diagnosed depression compared with antipsychotic users with Alzheimer's disease. Thus, possible reasons for antipsychotic use among the controls without Alzheimer's disease could be treatment of depression, anxiety or insomnia. Some of the controls without Alzheimer's disease could have dementia due to causes other than Alzheimer's disease such as vascular dementia. However, Alzheimer's disease is the most common form of dementia accounting up to $80 \%$ of the cases. ${ }^{35}$ Despite the use of antipsychotics among the controls, the findings of this study clearly show alarmingly high incidence of antipsychotic use among persons diagnosed with Alzheimer's disease.

In our study, risperidone was the most frequently initiated antipsychotic among persons with Alzheimer's disease. This is in line with the fact that risperidone is the only antipsychotic with approved indication for treatment of severe aggression in persons with Alzheimer's disease in Finland. A quarter of the users with Alzheimer's disease initiated antipsychotic use with quetiapine, although randomised controlled trials have not found evidence for the benefit of quetiapine use in the treatment of psychosis, agitation and global behavioural and psychological symptoms in dementia. ${ }^{19}$ Quetiapine is known to be used in the off-label treatment of insomnia ${ }^{40}$ and based on our clinical experience it is also frequently used for this indication in Finland which could 
explain the high rate of quetiapine use. However, antipsychotics should not be used in the treatment of insomnia. ${ }^{41}$

\section{Strengths and limitations of the study}

Important strengths of this study were the nationwide sample, clinically and imaging verified diagnoses of Alzheimer's disease and the ability to examine the incidence of antipsychotic use longitudinally over 12 years. A general limitation of Prescription Register data is that it is not known whether the dispensed drugs were actually taken. However, the validity of the Prescription Register in measuring exposure to antipsychotics among older people has been previously confirmed. ${ }^{42}$ The Prescription Register does not cover drugs used in hospitals and nursing homes which was taken into account by censoring follow-up at the start of long-term institutionalisation/hospitalisation. This may have underestimated the incidence of antipsychotic use as antipsychotic use is known to be higher in institutional settings. ${ }^{5}$ Further, we did not have information on the indication and appropriateness of antipsychotic use and the severity of BPSD and Alzheimer's disease. Persons with history of schizophrenia, schizotypal and delusional disorders or bipolar disorder were excluded to remove the effect of these diagnoses on the incidence of antipsychotic use. The strength of our study was the ability to compare the incidence of antipsychotic use among persons with Alzheimer's disease to the matched controls. Thus, the changes in incidence among persons with Alzheimer's disease are likely to be related to the symptoms associated with Alzheimer's disease as the incidence among the controls remained stable during the 12 years.

\section{Implications for clinical practice and research}

The high rate of antipsychotic initiations directly after Alzheimer's disease diagnosis is a concern as antipsychotics have been associated with an increased risk of serious adverse drug events. Instead of symptom-based treatment with antipsychotics there is a need for comprehensive clinical assessment to identify the underlying causes such as cognitive impairment. Future studies should assess the type and severity of BPSD for which antipsychotics are used around the time of diagnosis of Alzheimer's disease and whether the use is avoidable. Studying the feasibility and effectiveness of non-pharmacological approaches in representative cohorts is crucial to limit the use of antipsychotics only for most severe symptoms.

Marjaana Koponen, MSc, School of Pharmacy, University of Eastern Finland, Kuopio, Finland; Anna-Maija Tolppanen, PhD, Research Centre for Comparative Effectiveness and Patient Safety (RECEPS), University of Eastern Finland, Kuopio, Finland; Heidi Taipale, PhD, School of Pharmacy, University of Eastern Finland, Kuopio, Finland; Antti Tanskanen, Phil Lic, Department of Clinical Neuroscience, Karolinska Institutet, Stockholm, Sweden and National Institute for Health and Welfare, Helsinki, Finland; Jari Tiihonen, MD, PhD, Department of Clinical Neuroscience, Helsinki, Finland; Jari Tiihonen, MD, PhD, Department of Clinical Neuroscience,
Karolinska Institutet, Stockholm, Sweden, Department of Forensic Psychiatry, Karolinska Institutet, Stockholm, Sweden, Department of Forensic Psychiatry,
University of Eastern Finland, Niuvanniemi Hospital, Kuopio, Finland and National Institute for Health and Welfare, Helsinki, Finland; Kristina Johnell, PhD, Aging Research Center, Karolinska Institutet and Stockholm University, Stockholm, Sweden and Stockholm Gerontology Research Center, Stockholm, Sweden; Johan Fastbom MD, PhD, Aging Research Center, Karolinska Institutet and Stockholm University, Stockholm, Sweden; Riitta Ahonen, PhD, Sirpa Hartikainen, MD, PhD, School of Pharmacy, University of Eastern Finland, Kuopio, Finland

Correspondence: Marjaana Koponen, MSc, Faculty of Health Sciences, Schoo of Pharmacy, University of Eastern Finland, P.O. Box. 1627, FI-70211 Kuopio, Finland. Email: marjaana.koponen@uef.fi

First received 23 Dec 2014, accepted 9 Feb 2015

\section{Funding}

The research was funded by University of Eastern Finland Faculty of Health Sciences strategic funding. The funders had no role in study design; in the collection, analysis and strategic funding. The funders had no role in study design; in the collection, analysis and
interpretation of the data, in the writing of the report and in the decision to submit the manuscript for publication.

\section{References}

1 Lyketsos CG, Lopez O, Jones B, Fitzpatrick AL, Breitner J, DeKosky S Prevalence of neuropsychiatric symptoms in dementia and mild cognitive impairment: results from the cardiovascular health study. JAMA 2002; 288 : 1475-83.

2 Steinberg M, Shao H, Zandi P, Lyketsos CG, Welsh-Bohmer KA, Norton MC, et al. Point and 5-year period prevalence of neuropsychiatric symptoms in dementia: the Cache County Study. Int J Geriatr Psychiatry 2008; 23: 170-7.

3 Laitinen ML, Bell JS, Lavikainen P, Lönnroos E, Sulkava R, Hartikainen S. Nationwide study of antipsychotic use among community-dwelling persons with Alzheimer's disease in Finland. Int Psychogeriatr 2011; 23: 1623-31.

4 Calvo-Perxas L, de Eugenio RM, Marquez-Daniel F, Martinez R, Serena J, Turbau J, et al. Profile and variables related to antipsychotic consumption according to dementia subtypes. Int Psychogeriatr 2012; 24: 940-7.

5 Rattinger GB, Burcu M, Dutcher SK, Chhabra PT, Rosenberg PB, SimoniWastila $\mathrm{L}$, et al. Pharmacotherapeutic management of dementia across settings of care. J Am Geriatr Soc 2013; 61: 723-33.

6 Schneider LS, Dagerman KS, Insel P. Risk of death with atypical antipsychotic drug treatment for dementia: meta-analysis of randomized placebocontrolled trials. JAMA 2005; 294: 1934-43.

7 Gill SS, Bronskill SE, Normand SL, Anderson GM, Sykora K, Lam K, et al. Antipsychotic drug use and mortality in older adults with dementia. Ann Intern Med 2007; 146: 775-86.

8 Kales HC, Kim HM, Zivin K, Valenstein M, seyfried LS, Chiang C, et al. Risk of mortality among individual antipsychotics in patients with dementia. Am J Psychiatry 2012; 169: 71-9.

9 Rochon PA, Stukel TA, Sykora K, Gill S, Garfinkel S, Anderson GM, et al. Atypical antipsychotics and parkinsonism. Arch Intern Med 2005; 165: 1882-8.

10 Jalbert JJ, Eaton CB, Miller SC, Lapane KL. Antipsychotic use and the risk of hip fracture among older adults afflicted with dementia. J Am Med Dir AssoC 2010; 11: 120-7.

11 Gill SS, Rochon PA, Herrmann N, Lee PE, Sykora K, Gunraj N, et al. Atypical antipsychotic drugs and risk of ischaemic stroke: population based retrospective cohort study. BMJ 2005; 330: 445.

12 Wu CS, Wang SC, Gau SS, Tsai HJ, Cheng YC. Association of stroke with the receptor-binding profiles of antipsychotics - a case-crossover study. Biol Psychiatry 2013; 73: 414-21.

13 Livingston $\mathrm{G}$, Walker $\mathrm{AE}$, Katona $\mathrm{CL}$, Cooper $\mathrm{C}$. Antipsychotics and cognitive decline in Alzheimer's disease: the LASER-Alzheimer's disease longitudinal study. J Neurol Neurosurg Psychiatry 2007; 78: 25-9.

14 Vigen CL, Mack WJ, Keefe RS, Sano M, Sultzer DL, Stroup TS, et al. Cognitive effects of atypical antipsychotic medications in patients with Alzheimer's disease: outcomes from CATIE-AD. Am J Psychiatry 2011; 168: 831-9.

15 Rosenberg PB, Mielke MM, Han D, Leoutsakos JS, Lyketsos CG, Rabins PV, et al. The association of psychotropic medication use with the cognitive, functional, and neuropsychiatric trajectory of Alzheimer's disease. Int J Geriatr Psychiatry 2012; 27: 1248-57.

16 Lonergan E, Luxenberg J, Colford J. Haloperidol for agitation in dementia. Cochrane Database Syst Rev 2002; 2: CD002852.

17 Ballard C, Waite J. The effectiveness of atypical antipsychotics for the treatment of aggression and psychosis in Alzheimer's disease. Cochrane Database Syst Rev 2006; 1: CD003476.

18 Schneider LS, Dagerman K, Insel PS. Efficacy and adverse effects of atypical antipsychotics for dementia: meta-analysis of randomized, placebo-controlled trials. Am J Geriatr Psychiatry 2006; 14: 191-210.

19 Maher AR, Maglione M, Bagley S, Suttorp M, Hu JH, Ewing B, et al. Efficacy and comparative effectiveness of atypical antipsychotic medications for off-label uses in adults: a systematic review and meta-analysis. JAMA 2011; 306: 1359-69.

20 APA Work Group on Alzheimer's Disease and other Dementias, Rabins PV, Blacker D, Rovner BW, Rummans T, Schneider LS, et al. American Psychiatric Association practice guideline for the treatment of patients with Alzheimer's disease and other dementias. 2nd ed. Am J Psychiatry 2007; 164: 5-56.

21 Working group set up by the Finnish Medical Society Duodecim, Societas Gerontologica Fennica, the Finnish Neurological Society, Finnish Psychogeriatric Association and the Finnish Association for General Practice. Memory disorders. Current Care Guidelines, The Finnish Medical Society Duodecim, 2014 (http://www.kaypahoito.fi).

22 National Institute for Health and Clinical Excellence. Dementia: Supporting People with Dementia and their Carers in Health and Social Care. NICE clinical guideline 42. NICE, 2012.

23 Tolppanen AM, Taipale H, Koponen M, Lavikainen P, Tanskanen A, Tiihonen J, et al. Use of existing data sources in clinical epidemiology: Finnish health 
care registers in Alzheimer's disease research - the Medication use among persons with Alzheimer's disease (MEDALZ-2005) study. Clin Epidemiol 2013; 5: $277-85$

24 McKhann G, Drachman D, Folstein M, Katzman R, Price D, Stadlan EM. Clinical diagnosis of Alzheimer's disease: report of the NINCDS-ADRDA Work Group under the auspices of Department of Health and Human Services Task Force on Alzheimer's Disease. Neurology 1984; 34: 939-44.

25 American Psychiatric Association. Diagnostic and Statistical Manual of Mental Disorders (4th edn) (DSM-IV). APA, 1994.

26 WHO Collaborating Centre for Drug Statistics Methodology. The Anatomical Therapeutic Chemical Classification System. WHO Collaborating Centre for Drug Statistics Methodology, Norwegian Institute of Public Health, 2013. (http://www.whocc.no/atc_ddd_index/).

27 World Health Organization. The IDC-10 Classification of Mental and Behavioural Disorders: Diagnostic Criteria for Research. WHO, 1993.

28 World Health Organization. International Statistical Classification of Diseases and Related Health Problems, 9th Revision. WHO, 1975.

29 World Health Organization. International Statistical Classification of Diseases and Related Health Problems, 8th Revision. WHO, 1965.

30 Charlson ME, Pompei P, Ales KL, MacKenzie CR. A new method of classifying prognostic comorbidity in longitudinal studies: development and validation. J Chronic Dis 1987; 40: 373-83

31 Rosenberg PB, Mielke MM, Appleby BS, Oh ES, Geda YE, Lyketsos CG. The association of neuropsychiatric symptoms in $\mathrm{MCl}$ with incident dementia and Alzheimer disease. Am J Geriatr Psychiatry 2013; 21: 685-95.

32 Rockwood K, Cosway S, Carver D, Jarrett P, Stadnyk K, Fisk J. The risk of dementia and death after delirium. Age Ageing 1999; 28: 551-6.

33 Rahkonen T, Luukkainen-Markkula R, Paanila S, Sivenius J, Sulkava R. Delirium episode as a sign of undetected dementia among community dwelling elderly subjects: a 2 year follow up study. J Neurol Neurosurg Psychiatry 2000; 69: 519-21.

34 Davis DH, Muniz Terrera G, Keage H, Rahkonen T, Oinas M, Matthews FE, et al. Delirium is a strong risk factor for dementia in the oldest-old: a population-based cohort study. Brain 2012; 135: 2809-16.

35 Alzheimer's Association. Alzheimer's disease facts and figures. Alzheimers Dement 2014; 10: e47-92.

36 Martinez C, Jones RW, Rietbrock S. Trends in the prevalence of antipsychotic drug use among patients with Alzheimer's disease and other dementias including those treated with antidementia drugs in the community in the UK: a cohort study. BMJ Open 2013; 3: e002080.

37 Taipale H, Koponen M, Tanskanen A, Tolppanen AM, Tiihonen J, Hartikainen S. Antipsychotic doses among community-dwelling persons with Alzheimer disease in Finland. J Clin Psychopharmacol 2014; 34: 435-40.

38 Taipale $\mathrm{H}$, Koponen M, Tanskanen A, Tolppanen AM, Tiihonen J, Hartikainen S. Antipsychotic polypharmacy among a nationwide sample of communitydwelling persons with Alzheimer's disease. J Alzheimers Dis 2014; 41: 1223-8.

39 Inouye SK, Westendorp RG, Saczynski JS. Delirium in elderly people. Lancet 2014; 383: 911-22.

40 Hermes ED, Sernyak M, Rosenheck R. Use of second-generation antipsychotic agents for sleep and sedation: a provider survey. Sleep 2013; 36: 597-600.

41 Working group set up by the Finnish Medical Society Duodecim and the Finnish Sleep Research Society. Current Care Guidelines: Insomnia. 2015 (http://www.kaypahoito.fi).

42 Rikala M, Hartikainen S, Sulkava R, Korhonen MJ. Validity of the Finnish Prescription Register for measuring psychotropic drug exposures among elderly Finns: a population-based intervention study. Drugs Aging 2010; 27 : $337-49$. 